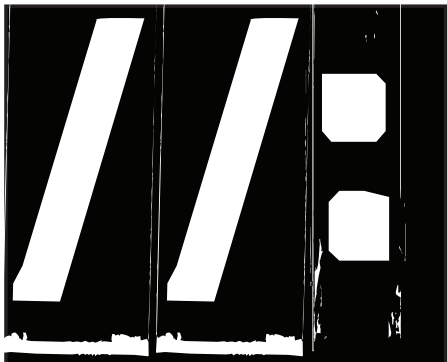

educäción y cömunicación

12: 88-104 Mayo 2016

\section{APORTACIONES DEL PROGRAMA ATAL A LOS GENTROS EDUCATIVOS DESDE UN PUNTO DE VISTA INTERCULTURAL}

\section{Contributions of the ATAL programme to the education centres from an intercultural point of view}

\author{
Cristina Goenechea Permisán \\ E.mail: cristina.goenechea@uca.es \\ Cristina Iglesias Alférez \\ E.mail: cristina.iglesias.alferez@gmail.com \\ Universidad de Cádiz (España)
}

\title{
Resumen:
}

En este trabajo analizamos la contribución del programa de Aulas Temporales de Adaptación Lingüística (ATAL en adelante) a la construcción de una escuela más intercultural en Andalucía. Se trata de una reflexión necesaria, dado que estas aulas son la principal respuesta del sistema educativo andaluz a la presencia de alumnado extranjero. Para ello, partimos del análisis de los resultados obtenidos en una investigación cualitativa (1) realizada durante el curso 2014-15 en la provincia de Cádiz, basada en entrevistas, grupos de discusión y observación participante. Esta información nos permitirá conocer la situación actual del programa en Cádiz, sus posibilidades y sus limitaciones y cómo esto repercute en las escuelas. Los principales aspectos sobre los que trabajaremos son organizativos (a qué alumnado atiende, cómo lo atiende y durante cuánto tiempo) y sobre el profesorado de estas aulas (formación, itinerancia entre los centros y funciones de su puesto).

Palabras clave: educación intercultural, ATAL, alumnado extranjero, Andalucía.

\section{Abstract:}

This study analyses the contributions of the programme Aulas Temporales de Adaptación Lingüística (Linguistic Adaptation Temporary Classrooms / Here in after ATAL) to the development of increasingly intercultural schools in Andalucia. This is a necessary reflection, since these classrooms are the main response by the Andalucía's education system to the presence of foreign students at school centres. For this purpose, we have started by analysing the results obtained from a qualitative research carried out during the academic year 2014-2015 in Cadiz province. It is based on interviews, discussion groups and the participative observation of the class groups by the researchers. The information obtained will allow us to recognize the current situation of the programme in Cadiz, its possibilities and limitations and how these may affect the schools. The main focuses of our research are going to be the organization (which students does it include, how does it care for them and for how long) and the teachers in those classrooms (training, itinerary employment between centres and functions).

Key words: intercultural education, ATAL, immigrant students, Andalucía

Recibido: 26/01/2015 Revisado: 29/03/2016 Aceptado: 19/04/2016 Publicado: 01/5/2016 


\section{IIA Interculturalidad, educación y comunicación}

\section{Introducción}

T a provincia de Cádiz constituye un enclave singular en relación con la confluencia de población extranjera. Por un lado, su posición geográfica como punto más cercano al continente africano define su papel de puerta de entrada a gran parte de la población inmigrante de origen magrebí y subsahariano. Los originarios de Marruecos representan un 21\% de los extranjeros empadronados (2) en 2014. Por otro lado, sus buenas condiciones climáticas y los bajos costes de productos y servicios están animando el asentamiento de una colonia de ciudadanos de la Unión Europea, principalmente británicos (5.810, un 13,5\% de los extranjeros empadronados en 2014) y alemanes (2.173, 5\% del total).

A lo anterior se une la presencia de inmigrantes centro-europeos (principalmente población rumana, con 3.262 , un $7,6 \%$ del total de extranjeros empadronados), y chinos $(1.849,4,3 \%)$ que al igual que ocurre en el resto de España, llegan aquí en busca de oportunidades laborales. En los últimos años se está incrementando también la población estadounidense, debido a la ampliación del personal militar destinado en la Base Naval de Rota. Parte de esta población se está asentando fuera de la propia Base, principalmente en las localidades de El Puerto de Santa María y Rota, aunque estos datos no se reflejan en el padrón municipal (3). La normativa que afecta al recurso educativo que es objeto de estudio en este trabajo es la Orden de 15 de enero de 2007, por la que se regulan las medidas y actuaciones a desarrollar para la atención del alumnado inmigrante y, especialmente, las Aulas Temporales de Adaptación Lingüística. Desde entonces no ha aparecido normativa nueva, aunque si se dan instrucciones anualmente. Las más recientes han hecho hincapié en la importancia de dar el apoyo dentro del aula y la limitación de centros a los que puede atender un docente de ATAL, estableciendo un máximo de cinco.

Respecto a su ubicación en la estructura organizativa de la Delegación de Educación, el programa ATAL depende del Gabinete de Compensatoria. La persona responsable de este servicio se ocupa en total de 19 programas de compensación educativa, que tienen en común en palabras suyas "la normalización de una dificultad de un niño", y que abarcan desde la atención domiciliaria hospitalaria a la atención en centros psiquiátricos, pasando por los centros de menores infractores, los centros de compensatoria, los centros que participan en el PROA (programa de refuerzo, orientación y apoyo), etc. El programa ATAL supone un grano de arena en el mar de la labor de la Administración Educativa andaluza. A pesar de su reducido tamaño, este recurso educativo llama la atención de numerosos investigadores, si nos atenemos a la abundante literatura científica existente sobre el tema.

En el curso 2014-15, 10 maestros atienden a 184 (4) alumnos "estrictamente ATAL" en 53 centros (32 CEIP y 21 IES) de toda la provincia de Cádiz. A estos 184 alumnos se suman otros 63 a los que se presta atención directa sin ser destinatarios de ese recurso según la normativa, con lo que el total asciende a 247. Estos 63 estudiantes -de los que más adelante hablaremos con más detalle- son registrados administrativamente y son alumnos ATAL a todos los efectos. Todos los docentes ATAL excepto uno -que solo tiene CEIPs- atienden tanto centros de Educación Primaria como Secundaria. La maestra que menos centros atiende trabaja en cuatro y la que más, en siete; siendo 
el promedio de cinco centros. El número de alumnos por profesor oscila entre 33 y 19, siendo 24 el término medio. La mayor parte del alumnado de ATAL en la provincia de Cádiz (63,6\%, 157 alumnos) cursa Educación Primaria; mientras que el $36,4 \%$ restante $(90$ alumnos) estudia ESO y PCPI. En el curso 2014-15 un total de 28 nacionalidades distintas están presentes en las escuelas gaditanas, siendo las más numerosas la marroquí, estadounidense, rumana y china, como podemos ver en la siguiente tabla:

Tabla 1: Nacionalidad de los estudiantes de ATAL en la provincia de Cádiz (curso 2014-15)

\begin{tabular}{|l|l|}
\hline Nacionalidad & $\mathbf{N}^{\mathbf{0}}$ alumnos \\
\hline Marroquí & 72 \\
\hline Estadounidense & 38 \\
\hline Rumana & 31 \\
\hline China & 33 \\
\hline Británica & 19 \\
\hline Brasileña & 11 \\
\hline Mauritana & 6 \\
\hline Polaca & 5 \\
\hline Pakistaní & 4 \\
\hline Húngara & 4 \\
\hline Rusa & 3 \\
\hline Filipina & 2 \\
\hline Nigeriana & 2 \\
\hline Alemana & 2 \\
\hline Senegalesa & 2 \\
\hline Rep. Centroafricana & 1 \\
\hline Kazaja & 1 \\
\hline
\end{tabular}

\begin{tabular}{|l|l|}
\hline Noruega & 1 \\
\hline Portuguesa & 1 \\
\hline Búlgara & 1 \\
\hline Letona & 1 \\
\hline Costamarfileña & 1 \\
\hline Italiana & 1 \\
\hline Francesa & 1 \\
\hline Turca & 1 \\
\hline Mozambiqueña & 1 \\
\hline Holandesa & 1 \\
\hline Lituana & 1 \\
\hline
\end{tabular}

Fuente: Elaboración propia a partir de datos facilitados por la Delegación de Educación

\section{Planteamiento metodológico}

Este trabajo se enmarca en el proyecto $\mathrm{I}+\mathrm{D}+\mathrm{I}$ "Construyendo diferencias en las escuelas, estudio de las trayectorias de las ATAL en Andalucía, de su profesorado y su alumnado" coordinado por el Prof. Javier García Castaño de la Universidad de Granada. Se trata de una investigación de corte cualitativo, cuyos métodos principales de recogida de la información son la observación participante y la entrevista. Esta investigación se propone observar cómo la puesta en marcha de dispositivos de atención especializada para la acogida en la escuela de cierta población, relacionada con la inmigración, han podido servir para el desarrollo de procesos de integración o para la implementación de formas de exclusión y/o de segregación. Busca comprender los procesos de diferenciación y las formas de 


\section{IIA Interculturalidad, educación y comunicación}

gestión de la diversidad cultural en la escuela.

En la provincia de Cádiz se han realizado dos estudios de caso: dos maestras de ATAL que atienden a 9 centros (5 y 4 respectivamente) durante el curso 2014-15, con un total de 38 (18 y 20) estudiantes extranjeros. La primera docente atiende en centros de Primaria y Secundaria mientras que la segunda, solo asiste a centros de Primaria. Los casos corresponden a las dos zonas principales de concentración de ATAL: uno se sitúa en el Campo de Gibraltar y otro en la Bahía de Cádiz. Los resultados que aquí se exponen están basados en un total de 18 entrevistas a tutores, jefes de estudios y directores. Se realizó también un grupo de discusión con todos los maestros ATAL de la provincia y entrevistas a la actual responsable del programa en la Delegación de Educación y a la que fue responsable durante los 7 años anteriores. También fueron realizadas unas 26 observaciones en los diferentes centros a los que asistían las docentes.

\section{Discusión de resultados}

Este apartado se encuentra dividido en una serie de dimensiones en forma de cuestiones surgidas del material que hemos obtenido a partir de las entrevistas, observaciones y grupo de discusión realizados durante el curso 2014-15. Éstas son las siguientes:

1. ¿A qué alumnado atienden los maestros de ATAL y durante cuánto tiempo?

2. ¿Es diferente su intervención en Primaria y Secundaria?

3. ¿Va más allá la función del maestro de ATAL o se limita a la enseñanza de la lengua en las horas que atiende al alumnado extranjero?

4. ¿Qué formación permanente reciben los docentes
ATAL y qué formación sobre interculturalidad tiene el resto del profesorado?

5. ¿Trabajan desde una perspectiva "intercultural" o meramente de enseñanza de la lengua?

6. ¿Cómo se organiza el ATAL? ¿El apoyo es inclusivo, dentro del aula, como prioriza la normativa?, ¿Son grupos estables?

Estas dimensiones nos ayudan a entender cuáles son las funciones de estos docentes, cómo trabajan y con quién. Lo que nos aporta información sobre las características de este programa y la de los profesores en los centros educativos a los que asisten.

\section{¿A qué alumnado atienden los maestros de ATAL y durante cuánto tiempo?}

Las ATAL están destinadas, según recoge la normativa en su artículo 7.1, a los alumnos extranjeros que no dominan la lengua vehicular de enseñanza y cursan entre $3^{\circ}$ de primaria y $4^{\circ}$ de la ESO. La filosofía que hay detrás de este planteamiento es la de entender que antes de esta edad los niños aprenden por inmersión, sin necesidad de recibir unas clases específicas de lengua para adquirirla. En la práctica, vemos que este es uno de los aspectos más controvertidos del planteamiento de estas aulas.

Si nos centramos en los datos del curso 2014-15, vemos que los docentes de ATAL atienden mayoritariamente a alumnado considerado "estrictamente ATAL" (es decir, comprendidos entre $3^{\circ}$ de primaria y $4^{\circ}$ de la ESO), pero también atienden a alumnado de otros cursos. Estos estudiantes de otros cursos no contabilizan a la hora de decidir a qué centro irá un docente, pero una vez que trabaja con ese centro la Administración apoya que se atienda también a ese alumnado. Al pa- 


\section{1/: Aportaciones del programa ATAL a los centros 11: educativos desde un punto de vista intercultural}

recer, este trabajo se valora de forma diferente, como se refleja en las palabras de una de las maestras entrevistadas (maestra de ATAL 1): "el trabajo que hago en el primer ciclo consta, pero es de segunda fila". Como ya hemos mencionado, en Cádiz se atiende a un total de 247 alumnos, 63 de los cuales no serían estudiantes ATAL si se aplicara la normativa de manera estricta. ¿Quiénes son esos 63 alumnos? resulta llamativo que 35 de ellos son estudiantes de $1^{\circ}$ o $2^{\circ}$ de primaria, no siendo este ciclo objeto de los ATAL. Parece que el inicio del uso de la lectoescritura hace que los centros y los docentes consideren más necesario acelerar de alguna manera el aprendizaje de la lengua que teóricamente debería producirse en esta etapa de manera natural, sin ayuda de clases específicas. Así recoge una maestra ATAL entrevistada (maestra de ATAL 1) su opinión respecto de la necesidad de atender a los alumnos de infantil y primer ciclo de primaria: "en el tema de infantil..., todos los estudios de aprendizaje de idioma apuntan o dicen que a esas edades se aprende de forma natural, igual que el idioma materno, con la exposición al idioma. Que hay que dar un tiempo, que son seis meses hasta que el alumno va hablando (...) Entonces, desde ese punto de vista, sacar a un alumno tan pequeño, donde en infantil lo que se debe hacer es jugar, participar con los compañeros y relacionarse mucho, sacarlo a darle una instrucción desde luego yo no soy partidaria. Otra cosa es que se asesore al profesor...(...). En el primer ciclo, ahí no estoy yo tan de acuerdo con la normativa... la normativa los ha quitado. Entonces desde el punto de vista del aprendizaje de la lengua todavía están en la edad. Hasta los ocho años se aprende de esta manera que te estaba contando, que es igual que el idioma materno, con una facilidad tremenda. Primero se van adquiriendo, van escuchando, y ya sobre los seis meses ya hablan casi perfectamente. Casi. Hay excepciones. Pero qué es lo que pasa, que ya empieza la lectoescritura en primero. Entonces, ahí si hay una...”.

Las opiniones no son unánimes en este punto. Así, la maestra que constituye nuestro segundo estudio de caso (maestra de ATAL 2) discrepa, no considera que deba atenderse en el ATAL a los niños y niñas de primer ciclo de primaria: "muchas veces cuando algunos profesores me plantean que por qué los niños de primero y de segundo no entran en el ATAL, precisamente les digo: es que resulta que las actividades que tienen en sus propios libros son muy visuales y además están aprendiendo a manejar todavía el idioma, entonces, realmente, no creo que haya que sacarlos tan pronto". Encontramos además esta opinión en una entrevista con el jefe de estudios del CEIP A, perteneciente a nuestro primer estudio de caso “(...) realmente, los más pequeños... no hay nada hecho para ellos porque no lo necesitan. Es decir, que dentro de la dinámica de la clase de infantil, terminan hablando más o menos, español. No perfectamente pero...".

Entre estos 28 alumnos no hay ninguno de Infantil, ningún ATAL atiende a menores de esta etapa. En una de las observaciones realizadas la investigadora anota una referencia al malestar existente en Infantil por la falta de recursos que tienen para atender a la diversidad: "la maestra me cuenta que en Infantil hay malestar porque los ATAL no atienden a este nivel, tampoco los logopedas, sienten que no tienen recursos" (CEIP C).

Recientemente la propia Consejería de Educación ha dado a conocer un informe sobre el funcionamiento de las ATAL (AGAEVE, 2015) en el que encuentra resultados similares a nivel andaluz. Así, cuando se le 


\section{IIA Interculturalidad, educación y comunicación}

pide al profesorado ATAL que dé su opinión acerca de cuándo debería comenzar este programa, la mayoría los docentes $(56,4 \%)$ cree que debería ser en $1^{\circ}$ de Primaria, el $13,5 \%$ cree que en $2^{\circ}$ de Primaria y el $18 \%$ piensa que en Educación Infantil. Tan sólo el 10,4 \% cree que debe comenzar en $3^{\circ}$ de Primaria como está estipulado actualmente.

La normativa también establece que el tiempo máximo que puede asistir un estudiante al ATAL es de un año y que sólo excepcionalmente podrán continuar asistiendo al finalizar este periodo. Parece que este es el caso de los 28 alumnos restantes de ese total de 63 registrados como "otros estudiantes de atención directa". Se trata de alumnos que están en los cursos que atiende oficialmente el ATAL (de $3^{\circ}$ de primaria a $4^{\circ} \mathrm{ESO}$ ) pero ya han completado su periodo máximo de estancia. ¿Quiénes son estos alumnos que necesitan un refuerzo más prolongado? si comprobamos su nacionalidad vemos que predominan los chinos (9) y los marroquíes (7), cuya lengua materna está más alejada del español. Menos numerosos son los rumanos (5), brasileños (2), y los procedentes de Reino Unido, Alemania, Lituania, Mozambique y EEUU con un alumno cada uno.

La literatura ya nos advierte que el tiempo necesario para aprender una lengua al nivel necesario para funcionar en ella académicamente es mucho mayor, siendo el periodo de un año totalmente insuficiente. Así Etxeberría y Elosegui (2010: 240) afirman siguiendo a Cummins que:

«los estudiantes inmigrantes pueden adquirir rápidamente una fluidez considerable en la lengua dominante, pero hace falta cinco años (y a menudo mucho más) para recuperar el terreno con los hablantes nativos en los aspectos académicos»
Los datos de otras investigaciones corroboran nuestras conclusiones en este sentido. Casi tres cuartas partes del profesorado de ATAL considera que la duración máxima del programa no es la adecuada y cerca del $72 \%$ tampoco cree adecuado el número de horas semanales que reciben. Más del $66 \%$ opina que lo conseguido tras la finalización del Programa ATAL no es suficiente para que el alumnado tenga acceso al currículo establecido (AGAEVE, 2015).

\section{¿Es diferente su intervención en Primaria y Secundaria?}

Según los datos a los que hemos tenido acceso a partir del material generado de nuestros dos estudios de caso, y sobre todo a través del segundo cuya docente (maestra ATAL 2), trabaja en centros de Primaria y Secundaria, observamos que la intervención en ambas etapas es similar. El material utilizado depende de la edad de los estudiantes así como de su nivel de español, por lo que todo el trabajo está centrado en las características individuales de cada persona incluso aunque la atención sea grupal. La docente trabaja aspectos lingüísticos en todos los niveles y tiene en cuenta el ritmo de aprendizaje de cada alumno, aunque en el caso de Secundaria intenta avanzar a un ritmo mayor para que tenga mayores oportunidades de trabajo en el aula ordinaria. Así nos explica la metodología que sigue: "cada alumno viene con un nivel. Si un alumno ya conoce los saludos, las despedidas, evidentemente no empiezo por ahí. Siempre busco lo más motivador posible y que enganche con el vocabulario que ellos puedan manejar (...). De hecho, muchas veces los alumnos que ya están un poco más avanzados, cuando tienen que hacer un trabajo de historia, 
me piden ayuda y yo se la doy. Es decir, "tengo que hacer un trabajo sobre esto", y claro, como están trabajando la lengua no me importa".

El mayor problema de trabajar en ambos niveles se produce al organizar el horario. Se intenta atender al mayor número posible de alumnos, esto es complejo ya que existen diferencias en la duración de las clases en Primaria y Secundaria. Esto es observado durante nuestra estancia con la ATAL del segundo caso y quedó recogido en la entrevista con la anterior Coordinadora Provincial, "normalmente el alumnado salía de su aula, procurando que fuese en el horario que menos le perjudicaba, y se atendía por el ATAL en grupo. Todo esto era más complicado en secundaria, por las características de organización, los horarios... de esta etapa".

En ambos estudios, se observa que los horarios de las docentes de ATAL se crean teniendo en cuenta el de los estudiantes para evitar las salidas en asignaturas que facilitan la interacción entre el alumnado autóctono y el extranjero, aunque esto no es siempre posible. Durante las observaciones realizadas en el segundo estudio, llama la atención que la relación en los centros de Primaria era más cercana que en la mayoría de los centros de Secundaria y aumentaba cuanto más pequeño era el centro educativo. Esto puede ser explicado por el tiempo que permanece el ATAL en los centros educativos y el número de profesorado trabajando en cada uno de ellos. En los centros de primaria se produce una mayor interacción con los tutores de los estudiantes extranjeros, el alumnado tiene un menor número de profesorado y es más sencillo coincidir con estos durante el tiempo que permanece el ATAL en el centro. En el caso de Secundaria se dificulta el intercambio y en la mayoría de institutos la relación del ATAL es más directa con la dirección y los orientadores.

La itinerancia del profesorado de este programa es un rasgo que dificulta la cercanía y el traspaso de información y materiales con el resto de profesorado. En ocasiones el profesorado de ATAL es un desconocido en los centros. Esto queda recogido en varias entrevistas informales con docentes de ATAL y en la entrevista con la anterior Coordinadora Provincial: "muchas veces había profesorado del centro que no sabía quiénes eran ni qué hacían allí".

La dificultad para encontrar un aula en la que atender a los estudiantes es prácticamente igual en los centros de Primaria que en los de Secundaria y la viven todos los docentes de ATAL de la provincia. La planificación del profesorado de ATAL tiene lugar una vez comenzado el curso, cuando saben el alumnado al que deberán de atender en un primer momento, esto provoca que los centros escolares tengan los espacios asignados ya a otras actividades. Por lo que este profesorado se encuentra atendiendo en espacios diversos $\mathrm{y}$ en muchos casos necesitando modificar sus actuaciones a partir de los recursos con los que cuentan cada día y en cada lugar. Esta situación no es agradable para las personas responsables de los centros educativos, que intentan ofrecer aquellos espacios libres aunque suponga cambiar de aula o espacio cada día que asiste al centro.

\section{¿Va más allá la función del maestro de ATAL o se limita a la enseñanza de la lengua en las horas que atiende al alumnado extranjero?}

Además de la atención directa al alumnado, la normativa establece entre las funciones del profesorado 


\section{IIA Interculturalidad, educación y comunicación}

de ATAL "facilitar al profesorado de los centros atendidos orientaciones metodológicas y materiales sobre la enseñanza del español como segunda lengua" (art. 9). Constatamos que en el horario semanal de estos docentes sólo hay dos horas destinadas a evaluación y asesoramiento. Antes permanecían durante ese tiempo en la sede del EOE pero desde el curso 2014-15 cuando no se les reclama desde ningún centro para realizar funciones de evaluación o asesoramiento, tienen clases programadas. De este modo, no queda ningún hueco en su horario para reuniones o coordinación. Hay que tener en cuenta que este profesorado aprovecha el recreo para desplazarse de un centro a otro por lo que las oportunidades de interaccionar con los tutores de un modo más informal son realmente escasas, limitándose a los encuentros fortuitos por el pasillo durante los cambios de clase. Las horas de permanencia por la tarde las realizan en el centro que ellos eligen, y acuden a otros centros a demanda de éstos.

También realizan tareas de evaluación y asesoramiento en centros a los que no atienden normalmente, situados en las localidades en las que trabajan. Así la maestra ATAL del primer estudio describe cómo se desarrolla esta parte de su labor: "el primer trimestre suelo tener más carga de este tipo de trabajo, después ya van llamando menos. No hay después ningún seguimiento ni nada, si no me llaman no voy. Echo en falta que no haya luego un seguimiento. Voy un día, evalúo, doy unas orientaciones, y luego no sé si han ido bien o no".

Los centros valoran muy positivamente esta labor del profesorado ATAL, que consideran fundamental para la acogida de este alumnado. Así lo describe la actual Coordinadora provincial del programa: "el problema de los alumnos inmigrantes es que cuando llegan, al centro que llegan, primero hay un agobio de: ¡no sé qué hacer! Después, hay una concienciación positiva. En general, el profesorado, y el equipo directivo dicen: "el niño no sabe, tenemos que hacer algo". La labor de ATAL no sólo es directa, hay una parcela importante que es el asesoramiento al profesorado y en la entrega de materiales. Entonces, los centros se sienten...cuando llega un niño muy agobiados, pero cuando tú le dices bueno pero va a ir un profesional a asesorarte, a dejarle materiales a tu profesorado, entonces, ya empieza la concienciación positiva". Esta responsable considera que sería bueno potenciar la formación de los docentes de ATAL para esta labor específica de asesoramiento. "Tienen mucha formación como docentes pero le falta formación como asesores, porque yo creo que los profesores del alumnado inmigrante tienen dos parcelas importantes: uno, la atención directa al alumno de enseñanza y otra parcela, que yo creo que es dónde menos están formados que es el asesoramiento al profesorado porque son la clave que pueden formar... Tú no puedes contratar un profesor para cada centro, pero tú puedes tener un técnico experto que asesore a otros y los enseñe".

Los jefes de estudio de los centros en los que realizamos los estudios de caso corroboran el valor que tiene para ellos la labor de asesoramiento de la maestra de ATAL: "nos ayuda mucho a cómo enfocar las actividades, cómo hacerlo" (Jefe de Estudios centro C), "también tenemos diez niños de infantil, ....se reúne con ellos (con los maestros de infantil) para decirle cómo tienen que hacer, qué material, por las tardes si eso viene [la maestra de ATAL]" (Jefe de Estudios centro D).

Esta labor de asesoramiento se constata también en 


\section{1/: Aportaciones del programa ATAL a los centros 11: educativos desde un punto de vista intercultural}

otras investigaciones realizadas a nivel autonómico. Así, recientemente se ha constatado que más de las tres cuartas partes de este alumnado utiliza materiales preparados por el profesorado de ATAL en las asignaturas impartidas en el aula ordinaria (AGAEVE, 2015).

\section{¿Qué formación permanente reciben los docentes ATAL y qué formación sobre interculturalidad tiene el resto del profesorado?}

En la selección al puesto de ATAL, se valora la experiencia previa de atención al alumnado extranjero, conocimiento de idiomas y formación en interculturalidad o cursos cuyo contenido esté relacionado con ella. Las personas que concursan para este puesto específico suelen ser maestros, nos cuenta la Coordinadora Provincial de Cádiz que "guardan más el perfil al puesto. Lo suelen solicitar más maestros que profesores en secundaria”.

Una vez como docente de ATAL, la oferta de cursos, jornadas o actividades que tienen lugar en Cádiz es bastante limitada y ha ido disminuyendo con los años. En los primeros años del programa, además de las reuniones anuales que unían a todos los docentes de ATAL de la provincia, existían diferentes cursos con los que complementar su formación de acceso al puesto y que permitían intercambiar recursos y experiencias con docentes de otras provincias andaluzas. Debido a la situación económica sobrevenida, comenzaron a descender el número de actividades ofertadas o las que se ofrecían ya habían sido realizadas con anterioridad y no eran del interés del profesorado y las últimas jornadas andaluzas tuvieron lugar hace dos cursos. Las reuniones anuales a principio y final de curso se siguen realizando, en estos encuentros los docentes de la provincia intercambian información y materiales.

Los profesores muestran su preocupación por la falta de actividades de formación, por ello mantienen el contacto entre ellos a través de un servicio de mensajería móvil, por el que comentan casos y solicitan ayuda a otros profesionales de ATAL. Otros docentes buscan participar en cursos en provincias cercanas a su localidad o crean blogs en los que abrir los recursos a otros profesionales de la educación.

No sólo es importante la formación del docente especializado, sino que el profesorado que trabaja en los centros educativos que cuentan con alumnado de otras procedencias en sus aulas, se forme en materia de interculturalidad. Los docentes de ATAL y docentes que trabajan con estudiantes que desconocen el español a los que hemos entrevistado, nos cuentan que en las escuelas en general, existe poca formación sobre cómo trabajar con este tipo de alumnado. A menudo los docentes del aula ordinaria se sienten desbordados por la diversidad, no sólo cultural sino de otros tipos, como recogemos en este testimonio de una de las maestras entrevistadas: "la realidad de un maestro, la mía, mi grupo de alumnos, mi tutoría, con mis casos de niños especiales que tengo, estas dos niñas de ATAL, esto es un guiso que... ¿cómo me puedo dedicar a todo el mundo al cien por cien?(...) Hay momentos en la mañana, que me siento desbordada, que no puedo atender a todos como yo quisiera. Y que no sé cómo hacerlo porque tengo a mi chico de PT que va por otro lado, mi grupo con todos los que van rápido y los que van lento, y mis dos niñas de ATAL que continuamente me están reclamando atención. Ya lo llevo mucho mejor, ya sé hacerlo mucho mejor. Pero al principio me he 


\section{IIA Interculturalidad, educación y comunicación}

visto así, como desbordada. Tantos frentes abiertos y yo sin saber a quién acudir al cien por cien porque tú quieres acudir a todos y no quieres dejar a nadie descolgado" (Tutora centro B). Muchos docentes reconocen que su formación en esta materia es nula o muy escasa, pero tampoco parece que la consideren prioritaria. Algunos se encuentran formándose actualmente en otros aspectos más cercanos a su titulación (por ejemplo, educación especial, matemáticas, etc.) y creen que la formación en interculturalidad es necesaria pero en un segundo nivel.

Los que sí han recibido cursos sobre interculturalidad en sus distintas vertientes los valoran de manera muy positiva. "Sí, se han hecho [cursos de esta temática]. Puedes ver que tengo en la puerta colocado en diferentes idiomas...Eso fue de un programa de interculturalidad que hubo que se hizo durante un par de años, mientras hubo dinero, cuando se acabó el dinero, se acabó el programa y se acabó todo. Y se notó. Porque se integraron muy bien y estuvieron muy bien e hicieron muchas actividades y sobre todo los de aquí intentaron conocer la cultura de los demás y quizás ese es el problema de que mucha gente no respete a los demás, porque no conozcan la cultura" (Director centro E).

Los docentes reclaman formación en idiomas para poder realizar mejor su trabajo. El jefe de estudios del centro D lo expone así, aunque matiza las condiciones que serían necesarias para asistir a esta formación: "lo que necesitamos es mejorar los idiomas. Lo que pasa es que, hay muchos que están dispuestos y otros que no, yo no estaría muy dispuesto. Si no me lo reconocen en jornada laboral o es una inmersión lingüística allí en Inglaterra o un campamento de verano o algo así que sea de inmersión. Es que estamos muy hasta arriba para eso". Respecto a las demandas formativas del profesorado ATAL, piden más capacitación en enseñanza del español para extranjeros. Textualmente, la maestra de ATAL de nuestro segundo caso nos dice: "no tenemos formación en ese aspecto (enseñanza del español para extranjeros), en que nos dieran... cómo trabajar el tema...y la lectoescritura también”. Esta docente cree que los docentes del aula ordinaria necesitan formación sobre cómo evaluar a un alumno que no domina la lengua. Este parece ser un asunto que genera inquietud, como se pone de manifiesto en el extracto de esta entrevista: "yo creo que ellos echarían en falta el hecho de que les prepararan un poco, que hubiera un poco de criterio de, al tener esos alumnos, saber cómo evaluarlos...porque ellos no saben, dicen "¿qué hago, lo suspendo?”. Ese es el principal problema, y yo también lo comprendo. ¿Qué haces con ese alumno? Si tú has visto que está trabajando, y que quiere trabajar pero no llega al nivel porque no tiene vocabulario, no tiene las herramientas para poder expresarse y aprender los contenidos".

\section{¿Trabajan desde una perspectiva intercultural o meramente de enseñanza de la lengua?}

El programa de ATAL tiene dos objetivos principales: dotar de lenguaje vehicular al alumnado que desconoce nuestro idioma y contribuir a la integración de éste a la vida del centro (art.6).

El primer objetivo está claro, nadie discute que la principal aportación del ATAL es contribuir significativamente a la mejora del nivel de lengua española de este alumnado. El dominio de la lengua es una necesidad inequívoca e imperiosa para los estudiantes recién llegados y por consiguiente para el centro que los recibe. De hecho, antes de existir el programa ATAL el apoyo a los extranjeros alófonos recién llegados era 
dado por otros profesionales. Sobre esto nos habla el Jefe de Estudios de uno de los colegios del primer caso, "necesitas un apoyo específico para estos alumnos porque si no cuando desconocen el idioma es muy difícil (...). Es decir, si no la tuviéramos realmente hubiéramos tenido que acudir pues al apoyo normal que tenemos, al profesor de compensatoria, a audición y lenguaje. Tradicionalmente se recurría al profesional de audición y lenguaje para que trabajase con ellos. Entonces, hubiéramos tenido que utilizar otro recurso (...) quitándoselo a otros alumnos (Jefe de estudios CEIP A).

Pero más allá de la lengua, parece que las ATAL suponen un apoyo muy importante en la integración de este alumnado, cumpliendo así el segundo objetivo de los planteados en la normativa. Según los resultados de la investigación que venimos citando (AGAEVE, 2015), todo el profesorado cree que el ATAL ha favorecido la integración escolar de su alumnado y más del $90 \%$ opina que contribuye al mantenimiento de la cultura de origen del alumnado extranjero.

En las entrevistas realizadas en nuestros dos estudios, las personas participantes nos explican que la función del docente de ATAL no se limita a enseñar la lengua, el principal objetivo de éstos es que el alumnado se integre. En palabras de la Coordinadora Provincial: “(...) lo que se hace es intentar que un alumno, cuando llega a un centro o cuando llega a un entorno o a un barrio, integrarlo dentro del centro, y luego darle el conocimiento de la lengua que, parece que es lo que hacemos primero". Ésta misma persona nos explica que este profesorado facilita la adaptación al medio, puesto que se convierte en la persona de referencia para los estudiantes.

En una de las observaciones la investigadora anota: "siguen trabajando el tema del deporte. Se para [la maestra ATAL] a explicarle lo que es un penalti, una falta etc. Me explica que lo hace porque también se tiene que integrar en el patio, no solo en clase".

En cuanto al mantenimiento de la cultura de origen del que habla el informe de la AGAEVE, podemos decir a través de las observaciones realizadas, que en el material que utilizan los docentes de ATAL, se intenta que el alumnado trabaje la lengua española a la vez que explican aspectos de su cultura y origen. Esto resulta muy atractivo cuando en el aula de ATAL hay niños y niñas de diferentes nacionalidades y hablan de sus tradiciones, fiestas, comidas típicas, lo que le gusta de su país. Esto depende también del nivel del alumnado, como explica la maestra de ATAL del segundo estudio: "si un alumno ya conoce los saludos, las despedidas, evidentemente no empiezo por ahí. Siempre busco lo más motivador posible y que enganche con el vocabulario que ellos puedan manejar (...). Por ello, siempre intento partir de su contexto, y claro, cuando es posible el ordenador me ayuda mucho. La lectoescritura, claro que hay que trabajarla, pero intento que no todo sea lectoescritura porque es un poco aburrido y tampoco es todo mi cometido, lo que nosotros tenemos que trabajar es que aprenda a comunicarse; que mejore su capacidad de comunicación (...). Si tengo un grupito a veces he utilizado un método (...), “el mañana", que engloba todas las destrezas, y entonces dentro del mismo método trabajamos la comprensión y expresión escrita y oral, y hacen actividades grupales en las que tienen que interaccionar".

En ocasiones, los docentes buscan ayuda de aplicaciones móviles, traductores o materiales online con el que apoyar las explicaciones sobre todo cuando el alumnado acaba de llegar.

La anterior Coordinadora Provincial de Cádiz reconoce que la perspectiva intercultural está poco presente 


\section{IIA Interculturalidad, educación y comunicación}

en los centros y que se limitan a enseñar la lengua al alumnado recién llegado y no creen necesario por regla general realizar cambios más profundos. Así, nos explica que: "es poco frecuente [la perspectiva intercultural]. Hombre, la lengua sí, el enseñarles el español eso está claro, o con el ATAL, o con el profesor de apoyo, eso sí que se organiza porque claro, es una necesidad prioritaria para que el alumno empiece a aprender, eso es algo que les preocupa. Pero ya el tema de interculturalidad de que nosotros también tenemos que conocer algo de este niño, de dónde viene, de qué es lo que hace, qué es lo que ha hecho hasta ahora, qué es lo que ha comido hasta ahora, cómo nos ve... eso no, no es muy frecuente".

\section{¿Cómo se organiza el ATAL? ¿El apoyo es inclusivo, dentro del aula, como prioriza la normativa?, ¿Son grupos estables?}

En el curso 2014-15 en la provincia de Cádiz, los ATAL asistían a una media de 5 centros educativos. Esta situación hace que el profesorado tenga un horario muy apretado y repartido de manera que sean apoyados el máximo de estudiantes posible. Para que esto se pueda llevar a cabo, en la mayoría de los centros el ATAL debe agrupar al alumnado, y el grupo de estudiantes pueden o no tener el mismo nivel de español y ser de diferentes nacionalidades. Esto hace que el docente de ATAL deba ser flexible y adaptar el material dependiendo de cada estudiante.

La escasez de tiempo en los horarios de este profesorado, las diferentes situaciones de los centros educativos, así como el número de alumnado y los cursos en los que están matriculados hace prácticamente imposible el trabajo del ATAL dentro del aula ordina- ria. Como nos explica la Coordinadora Provincial del programa, "un ATAL tiene aquí cinco niños de cinco cursos diferentes y no va a dejar cuatro para entrar con el de primero de primaria. (...) Hay centros que se puede, centros que no". La orientadora del centro D refuerza esta postura: "Es más fácil que salgan (...). Es la forma por organización, por disponibilidad de recursos temporal es lo que podemos hacer, que salgan de clase, es que no nos queda otra".

Durante el curso de nuestra investigación de los 10 docentes de ATAL de la provincia sólo dos de ellos entran en el aula ordinaria, aunque esta atención se limita a dos centros educativos, como nos explican durante el grupo de discusión mantenido. Por tanto, el apoyo dentro del aula es absolutamente minoritario, dándose en 2 de los 53 centros que cuentan con ATAL este curso. Podemos afirmar que en la provincia de Cádiz no se cumple la recomendación establecida en la normativa de dar el apoyo dentro del aula. "El apoyo dentro del aula no se ha podido llevar a cabo por falta de tiempo" (Anterior Coordinadora Provincial).

Esta realidad es constatada a nivel andaluz en otras investigaciones (AGAEVE, 2015), siendo tan sólo un $9,1 \%$ del alumnado los que reciben atención en el aula ordinaria, mientras que una abrumadora mayoría del $90,9 \%$ lo hace en grupos de apoyo fuera del aula ordinaria.

Destacamos que en las escasas ocasiones en que se da apoyo dentro del aula éste es valorado de manera muy positiva tanto por la docente del aula ordinaria como por la de ATAL.

Por otra parte, en los dos estudios de caso realizados las docentes tienen unas dos sesiones por semana y estudiante, por lo que la mayoría de alumnos reciben 4 horas semanales de apoyo ATAL. Esto no es del todo 


\section{I/: Aportaciones del programa ATAL a los centros 11: educativos desde un punto de vista intercultural}

suficiente y es por ello que muchos de los estudiantes necesitan atención por otro tipo de especialista que le ayude en el aprendizaje del idioma o refuerce la lectura o escritura. Esto quizás no sería necesario si el profesorado ATAL asistiera a menos centros y pudiera permanecer más tiempo en los centros educativos, trabajando directamente con el alumnado o realizando una mayor labor de asesoramiento con los tutores y docentes que trabajan con estos estudiantes.

Las dificultades para organizar los horarios del profesorado de ATAL no acaban a principios de curso, ya que el alumnado puede llegar o marcharse a otra provincia o incluso país en cualquier momento del curso escolar, lo que supone replantear y planificar de nuevo el horario de los docentes. Los cambios no se limitan a los alumnos de un centro sino que en ocasiones conlleva variaciones en los centros a los que atiende el docente a lo largo del año.

En la entrevista mantenida con la Coordinadora Provincial de Educación Compensatoria, nos explicaba que la planificación del tiempo de cada maestra de ATAL para el curso escolar actual, habían sido modificada hasta en cinco ocasiones. La población extranjera presenta una movilidad muy alta y esto trae una mayor inestabilidad a la labor de los docentes de las ATAL. No cabe duda que la flexibilidad es una de las principales cualidades de este programa, que es capaz de adaptarse a las necesidades de los centros educativos.

En ocasiones, aunque se intente modificar dicho planning, no es posible por falta de tiempo y estos estudiantes entran en una lista de espera para ser atendidos lo antes posible. Entretanto, el docente asesora e intercambia materiales para que el estudiante comience con el apoyo.

\section{Conclusiones}

A partir del trabajo de campo realizado en esta investigación, hemos podido conocer la situación del programa ATAL en nuestra provincia. A continuación recogemos las principales conclusiones alcanzadas:

Hay dos aspectos recogidos en la normativa que parecen no cumplirse por no adaptarse a la realidad del programa y las necesidades de los centros y los alumnos. Se trata del apoyo inclusivo dentro del aula y la edad de incorporación al programa ATAL.

El apoyo dentro del aula constituye la excepción y no la norma, al contrario de lo que establece la Orden de 15 de enero de 2007. En la provincia de Cádiz el apoyo dentro del aula es absolutamente minoritario, dándose tan sólo en 2 de los 53 centros que cuentan con ATAL este curso. Aparecen distintos argumentos para explicar esta realidad, destacando con fuerza el poco tiempo de que dispone el profesorado para atender cada centro, que hace necesario sacar a los alumnos de sus respectivas aulas para atenderles de manera conjunta.

Con frecuencia se atiende también a niños y niñas de $1^{\circ}$ y $2^{\circ}$ de Primaria, que no son destinatarios del programa ATAL según la normativa. La mayor parte de los profesores opinan que los alumnos del primer ciclo de Primaria deberían ser también destinatarios de este recurso. La necesidad de atenderles se justifica por el inicio del uso de la lectoescritura y el comienzo de procesos de evaluación más sistemáticos a estas edades.

El programa ATAL no sólo supone enseñanza de la lengua sino que también realiza una importante labor en la integración del alumnado extranjero y en el asesoramiento al profesorado del aula ordinaria sobre cómo trabajar con ellos. 


\section{IIA Interculturalidad, educación y comunicación}

Uno de los principales problemas es la falta de formación en interculturalidad del profesorado en general, que provoca que se vea desbordado cuando se enfrenta a un aula diversa en la que la metodología no permite ofrecerle a cada alumno lo que realmente necesita. Además, aunque en el Plan de Acción Tutorial de los centros suele estar previsto cómo atender y acoger al alumnado que carece del español como lengua vehicular, carecen de un plan específico para atender a este alumnado, por lo que la presencia de los profesionales de ATAL y su asesoramiento es muy necesario en las escuelas y consideramos que el programa contribuye a una escuela más intercultural. Puesto que la formación de los docentes no es la suficiente para atender en el aula ordinaria a este alumnado, necesitan la ayuda de un docente especializado que le dirija en los primeros momentos y aconseje sobre qué material y metodología utilizar en el aula para que pueda participar estos alumnos y alumnas en el aula ordinaria. Si no existiera el programa ATAL, los centros educativos tendrían que asignarle esta tarea a los profesionales de apoyo a la integración que estuvieran en el centro. Es muy importante tener en cuenta que los recursos son muy limitados y no contar con ellos influye de forma directa en el desarrollo educativo de los estudiantes.

El futuro de las ATAL en incierto puesto que el número de docentes ha ido descendiendo en los últimos años, mezclándose entre las razones una menor llegada de población extranjera y una disponibilidad de recursos mucho menor. Hace falta indagar -como plantea la investigación en la que se inscribe este artículo- las trayectorias del alumnado ATAL. Los resultados de este alumnado se producen muy a largo plazo y en ocasiones, por el abandono de la escuela o del país, se desconoce su situación académica. Esto hace que los casos de éxito académico de este alumnado sean difíciles de localizar, lo que no quiere decir que no existan.

Los centros educativos valoran muy positivamente la existencia de este recurso y todas las personas consultadas consideran que es necesario en las escuelas que cuentan con alumnado que desconoce el idioma y reclaman poder contar con este profesorado más tiempo.

Notas

(1) Proyecto I+D+i titulado "Construyendo diferencias en la escuela. Estudios de las trayectorias de las ATAL en Andalucía, de su profesorado y de su alumnado" (Ref. CSO2013-43266-R), dirigido por F. Javier. García Castaño. Los miembros del equipo de investigación que participan en la provincia de Cádiz son: Ramón Porras Vallejo, Rafael A.Jiménez Gámez, Cristina Iglesias Alférez y Cristina Goenechea Permisán.

(2) Datos del padrón municipal disponibles en la web del INE.

(3) Recientemente se ha publicado que en Rota hay 963 casas alquiladas por estadounidenses y en El Puerto de Santa María 868. Rota al Día 21/03/2016. Disponible en: http://rotaaldia.com/not/16712/rotacuenta-en-la-actualidad-con-963-casas-alquiladas-amilitares-norteamericanos/

(4) Datos facilitados por la Delegación de Educación 


\section{I/: Aportaciones del programa ATAL a los centros 1. educativos desde un punto de vista intercultural}

\section{Referencias}

AGAEVE, Agencia Andaluza de Evaluación Educativa (2015). Estudio sobre la organización y el funcionamiento de las aulas temporales de adaptación lingüística (ATAL). Sevilla: autor- Consejería de Educación.

Del Río Fernández, J.L. (2015). Evaluación de las posibilidades educativas del aula ATAL en los centros escolares de Málaga. Estudio de casos. Universidad de Málaga, Tesis Doctoral. Disponible en: http:// riuma.uma.es/xmlui/bitstream/handle/10630/10575/ TD_Del_Rio_Fernandez.pdf?sequence $=1$

Etxeberría, F., y Elosegui, K. (2010). Integración del alumnado inmigrante: obstáculos y propuestas. Revista Española de Educación Comparada, (16), 235-264. Recuperado el 20 de abril de 2015 de http://revistas. uned.es/index.php/REEC/article/view/753.

García Castaño, F. J., Castilla Segura, J., \& Capellán de Toro, L. (2015). ¿Compensar es igualar?: trayectorias de escolares de nacionalidad extranjera en dispositivos "especiales" en el sistema educativo andaluz. In F. J. García Castaño, A. Megías Megías, \& J. Ortega Torres (Eds.), Actas del VIII Congreso sobre Migraciones Internacionales en España (Granada, 16-18 de septiembre de 2015) (pp. S02/13-S02/28). Granada: Instituto de Migraciones.
Jiménez Gámez, R.A. (Coord.) (2009). Estudio de la atención educativa prestada al alumnado inmigrante en las Aulas Temporales de Adaptación Lingüística en Cádiz. Informe de Investigación. Recuperado de http://minerva.uca.es/publicaciones/asp/docs/tesis/ cotrina $\% 20$ garc $\% \mathrm{C} 3 \% \mathrm{ADa} \% 20 \mathrm{y} \% 20$ otros.pdf http://revistas.uned.es/index.php/REEC/article/ view/7531

Moscoso García, F. (2013). El programa hispano-marroquí de enseñanza de Lengua Árabe y Cultura Marroquí (LACM) sometido a revisión. Árabe marroquí y amazige, lenguas nativas (L1). Anaquel de Estudios Árabes, 24, 119-135.

Orden de 15 de enero de 2007, de la Consejería de Educación de la Junta de Andalucía, por la que se regulan las medidas y actuaciones a desarrollar para la atención del alumnado inmigrante y, especialmente, las Aulas Temporales de Adaptación Lingüística. (BOJA núm.33, 14-02-2007).

Porras Vallejo, R., García García, M., y Cotrina García, M. (2009). Posibilidades y límites del programa de ATAL en el marco de una escuela inclusiva. El caso de la provincia de Cádiz. Revista de Educación inclusiva, 2(1), 11-28. Recuperado de http:/www.ujaen.es/ revista/rei/linked/documentos/documentos/2-1.pdf 